\title{
INFECTION
}

\section{Trichomonas infection influences the vaginal microbiome}

Trichomonas vaginalis (TV) infection is known to be associated with a number of serious long-term sequelae, including pelvic inflammatory disease and acquisition of HIV. Studies have shown that the microbiological environment of the vagina can interact with TV: preexisting bacterial vaginosis (BV-defined by Nugent Score [NS]) is a risk factor for TV infection, and antibacterial treatment of TV in HIV-positive women with BV is less effective than in those without BV. These data suggest that the pre-existing vaginal microbiota can offer a protective environment for TV in the vagina. Now, a new study has shown that TV infection is strongly associated with the vaginal microbiotic environment, which is altered in women with TV infection, possibly influenced by TV itself for its own benefit.

The study, led by Prof. David Martin, involved microbiota analyses on 60 women. The presence of STIs, including TV, was evaluated and NS was calculated by an experienced technician blinded to the patients' microbiota analysis results.

\section{$44 . . . T V$ takes on the role of a farmer in the vaginal environment by cultivating a flora that better suits its own purposes 77}

$30 \mathrm{TV}$-positive women were matched with $30 \mathrm{TV}$-negative women.

Vaginal microbiota analyses identified 122 operational taxonomic units (OTUs) in the study participants, with increased prevalence of specific OTUs in TV-positive women compared with TV-negative women. "Subcluster A1 microbiota, identified for the first time in our paper, are found only in the presence of TV and are associated with an enhanced host inflammatory response." Martin told Nature Reviews Urology, "vaginal inflammation is a major risk factor for HIV susceptibility and transmission."

Interestingly, another subcluster of vaginal microbiota identified as significantly more prevalent in women with TV (subcluster A3) was characterized by a previously unknown Mycoplasma strain. Dubbed 'Mnola' after New Orleans, LA, where the team is based, it is possible that this strain could prove to be a new species. Another species, M. hominis, has a symbiotic relationship with TV, but is also found in TV-negative women and is, therefore, not an obligate symbiont. Mnola, however, might be-all but one of the women in whom it was found were TV positive.

TV infection seems to cause a shift in the vaginal microbiota, towards subclusters A1 and A3. As Martin comments: "we think that TV takes on the role of a farmer in the vaginal environment by cultivating a flora that better suits its own purposes."

Annette Fenner

Original article Martin, D. H. et al. Unique vaginal microbiota which include an unknown Mycoplasma-like organism are associated with Trichomonas vaginalis infection. J. Infect. Dis. doi:10.1093/infdis/jit100 